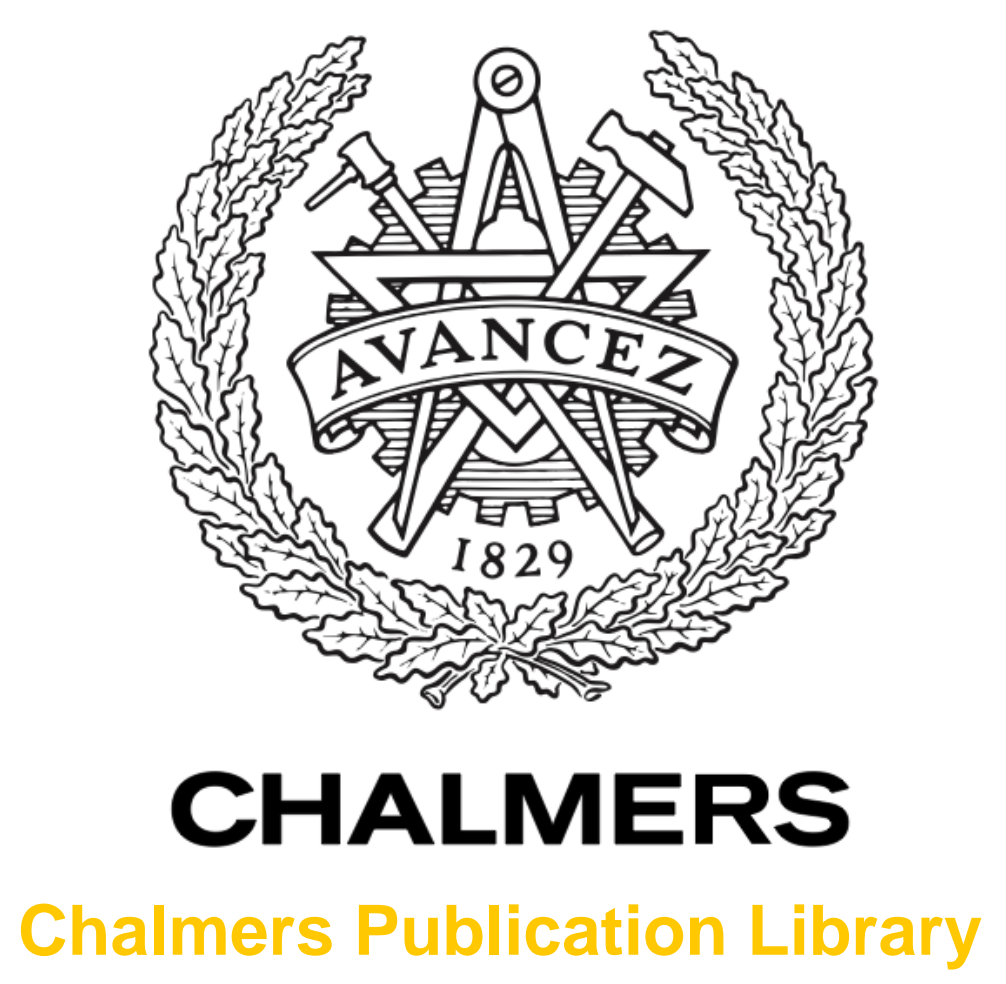

\author{
Mitigation of Nonlinear Impairments on QPSK Data in Phase-Sensitive Amplified \\ Links
}

This document has been downloaded from Chalmers Publication Library (CPL). It is the author's version of a work that was accepted for publication in:

39th European Conference on Optical Communication and Exhibition, ECOC 2013, London, 22-26 September 2013

Citation for the published paper:

Corcoran, B. ; Olsson, S. ; Lundström, C. (2013) "Mitigation of Nonlinear Impairments on QPSK Data in Phase-Sensitive Amplified Links". 39th European Conference on Optical Communication and Exhibition, ECOC 2013, London, 22-26 September 2013

http://dx.doi.org/10.1049/cp.2013.1435

Downloaded from: http://publications.lib.chalmers.se/publication/192418

Notice: Changes introduced as a result of publishing processes such as copy-editing and formatting may not be reflected in this document. For a definitive version of this work, please refer to the published source. Please note that access to the published version might require a subscription.

Chalmers Publication Library (CPL) offers the possibility of retrieving research publications produced at Chalmers University of Technology. It covers all types of publications: articles, dissertations, licentiate theses, masters theses, conference papers, reports etc. Since 2006 it is the official tool for Chalmers official publication statistics. To ensure that Chalmers research results are disseminated as widely as possible, an Open Access Policy has been adopted.

The CPL service is administrated and maintained by Chalmers Library. 


\title{
Mitigation of Nonlinear Impairments on QPSK Data in Phase-Sensitive Amplified Links
}

\author{
Bill Corcoran, Samuel L.I. Olsson, Carl Lundström, Magnus Karlsson, Peter A. Andrekson
}

Fiber Optic Communications Research Center (FORCE), Photonics Lab., Dept. Microtechnology and Nanoscience, Chalmers University of Technology, Gothenburg, Sweden, billc@chalmers.se

\begin{abstract}
We investigate the mitigation of nonlinear impairments via phase-sensitive amplification. We show in simulation and experiment that this effect can be optimized through engineering link dispersion. A phase-sensitive amplified link is measured to reduce nonlinear penalties by over $3 d B$ compared to a phase-insensitive amplified link.
\end{abstract}

\section{Introduction}

Phase-sensitive amplifiers (PSAs) are known to theoretically provide amplification with a $0 \mathrm{~dB}$ noise figure - ideally noiseless amplification. To date, PSAs for optical systems have exploited optical nonlinearities in $\chi^{(2)}$ or $\chi^{(3)}$ materials ${ }^{1,2}$. In linear systems, PSAs have been shown experimentally to enable improved performance in optical communication links ${ }^{3,5}$. The phasesqueezing properties of PSAs has also been used to reduce the impact of nonlinear phase noise for some specific modulation formats ${ }^{4,5}$. However, in PSA systems capable of modulation format independent operation, the consequences of nonlinear phase noise have yet to be understood.

A PSA system capable of modulation format independent operation, called a 'copier-PSA', consists of two cascaded fiber-optic parametric amplifiers (FOPAs) ${ }^{1}$. In this system, the first FOPA creates a phase conjugate copy of the signal at the idler wavelength. If the output signal (S), idler (I) and pump waves are all transferred via an optical link to the input of the second FOPA, this amplifier operates as a PSA. In the small-signal regime the PSA essentially performs a coherent summation of the signal and the conjugate of the conjugate idler (i.e. $\left.\mathrm{S}_{\mathrm{PSA}}=\mu \mathrm{S}+v l^{*}\right)^{1}$. In the linear transmission regime, this is equivalent to summing the signal with a copy of itself (i.e. $I=S^{*}$ ). In the nonlinear transmission regime, the signal and idler are distorted in phase. While uncorrelated distortions would result in amplitude distortions via phase-sensitive (PS) gain, if the distortions in phase are correlated on the signal and idler, their summation can cancel these distortions.

If both signal and idler propagate in a dispersion-less channel, the nonlinear distortion experienced by each will be correlated. However, if the signal and idler undergo dispersion, each will be affected differently resulting in uncorrelated nonlinear distortions that will not be cancelled by coherent summation. As such, it is important to try to quantify the impact dispersion will have on the mitigation of nonlinear distortion, which has not been shown previously ${ }^{6,7}$.

Here we investigate the mitigation of nonlinear phase distortions enabled by the coherent summation property of PSA. We numerically investigate nonlinear distortion of QPSK data in a dispersive standard single mode fiber (SSMF) link, finding that in a copier-PSA system there is an optimal dispersion map enabling the greatest reduction of nonlinear distortions. We then validate this effect through experiment, measuring a $3 \mathrm{~dB}$ decrease in nonlinearityinduced Q-penalty in the PS system compared with a phase-insensitive (PI) system.

\section{Concept and Simulations}

The concept behind the mitigation of nonlinear phase distortions through coherent summation is shown in Fig. 1. The initial (a) signal (red) and conjugated signal copy (i.e. idler, green) are nonlinearly distorted in phase similarly through transmission (b). When summing the transmitted signal with the conjugate of the copy (c), the phase distortion can then be squeezed at the expense of some amplitude noise. It can be shown that if the phase distortion $(\delta \varphi)$ on the signal and copy are correlated, then the resulting amplitude distortion $(\delta A)$ after summation grow as $\delta A=2 \cos (\delta \varphi)$, so the mapping of phase-to-amplitude amplitude distortions is $<1: 1$ for deviations $\pm \pi / 4$. This property has been exploited recently to mitigate nonlinearities in the electronic domain after coherently detecting the transmitted fields ${ }^{6,8}$, in the case of PSA this summation occurs alloptically and helps explain the observed behavior of a copier-PSA system in the nonlinear transmission regime ${ }^{7}$.

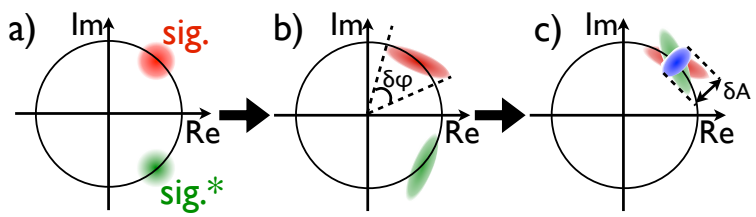

Fig. 1: Concept of mitigation of nonlinearity through coherent superposition. a) Before transmission, b) after transmission and c) after superposition. 


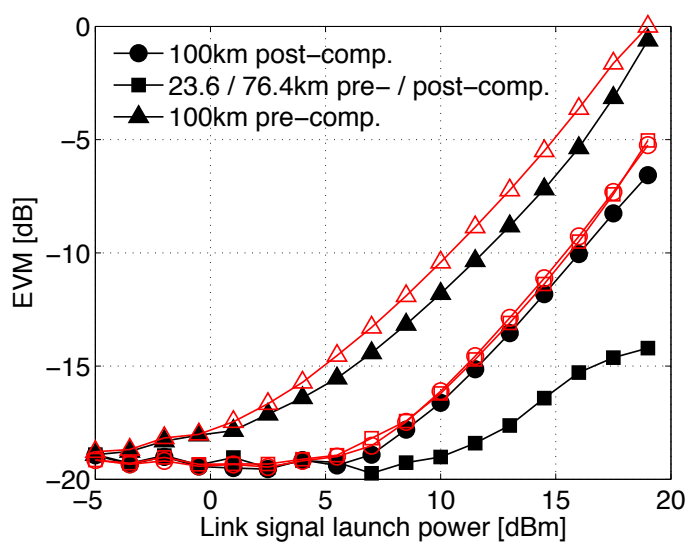

Fig. 2: Simulated EVM results with (solid black PS) and without (open red - PI) superposition of signal and conjugate copy. Legend indicates the dispersion scheme used.

In our simulations, we model an ideal $10 \mathrm{Gbd}$ QPSK signal numerically using 200 samples per bit, with a $16 \mathrm{GHz}$ modulator emulated by a 5th order Bessel filter. The OSNR of the signal is set at $40 \mathrm{~dB}$, which allows the effects of dispersion in the system to be highlighted over noise driven effects. In our copier-PSA system, the signal pump and idler wavelengths are typically spaced by $>8 \mathrm{~nm}$, as such nonlinear cross-talk should be negligible allowing us to treat signal and idler propagation as independent. The copier-PSA system under investigation is single polarization, so we use a standard single polarization nonlinear Schrödinger equation to model propagation, solved with a split-step Fourier method algorithm. The copier is emulated by simply conjugating the generated signal field and the PSA emulated after propagation by coherently summing the signal and idler fields. The link parameters used are for a $100 \mathrm{~km}$ span of SSMF. Since the coherent summation in the PSA happens all-optically, the link must be fully dispersion compensated. We model this as a single dispersion step without nonlinearity. To allow for reasonable computation time the output field error-vector magnitude (EVM) is used as a metric for comparison instead of true bit-error rate (BER).

Fig. 2 shows EVMs of the transmitted signal against signal link launch power with and without coherent summation corresponding to $\mathrm{PI}$ and PS amplification. Three separate dispersion maps are used; full pre-dispersion, full postdispersion and an optimized balance between pre- and post-dispersion. This optimum was determined from a parameter sweep of varied pre- and post- compensation values. Comparing operation in either PI or PS mode for both the fully pre- and post- compensated models, we see that the PS case shows marginal EVM improvement compared to the PI case. However for the case where $23.6 \mathrm{~km}$ pre-compensation is applied, the improvement of EVM comparing PI and PS is more pronounced. It is also worth noting that the earlier onset of nonlinear distortion (irrespective of PI or PS operation) for the full pre-compensation case than for the full post- or optimized cases. This is likely the product of a higher level of dispersion-induced inter-symbol interference in the fully precompensated case.

\section{Experiments}

The implemented copier-PSA system is shown in Fig. 3, based on previous demonstrations ${ }^{3,7}$. A 10GBd QPSK signal at $1545.3 \mathrm{~nm}$ and phasemodulated pump at $1553.7 \mathrm{~nm}$ are mixed in the copier FOPA. A wavelength division multiplexing (WDM) coupler then separates pump from signal and idler, the pump is attenuated (for $10 \mathrm{dBm}$ link launch power) and the signal and idler passed through and optical processor (OP Finisar WaveShaper) for delay and amplitude trimming $^{3,7}$. The signal and idler are then amplified by an EDFA and then attenuated to control launch power into the link. After the link, the pump is recovered using a hybrid EDFA / injection-locking system ${ }^{3,7}$. Signal, pump and idler are then mixed in the second FOPA, set (by tuning pump power) to provide $21 \mathrm{~dB}$ on-off gain in either $\mathrm{PI}$ or $\mathrm{PS}$ mode (selected by blocking or passing the idler through the OP). The amplified signal is then passed through a 1bit delay interferometer before an amplified, balanced receiver. The differential QPSK pattern is then analyzed. Both FOPAs used are designed to suppress stimulated Brillouin scattering $^{9}$. In the pre- and post-compensated systems, the link is comprised of a fiber Bragg grating dispersion compensating unit (FBG$\mathrm{DCM}$ - Proximion), placed at point $\mathrm{B}$ or $\mathrm{C}$, and $81.4 \mathrm{~km}$ SSMF. In the optimized case a $105 \mathrm{~km}$ SSMF span is pre-compensated with a $23.6 \mathrm{~km}$ equivalent dispersion-compensating fiber (DCF) unit placed before the signal/idler booster EDFA (A) to avoid nonlinear effects in the DCF, and post-compensated with the FBG-DCM (C).

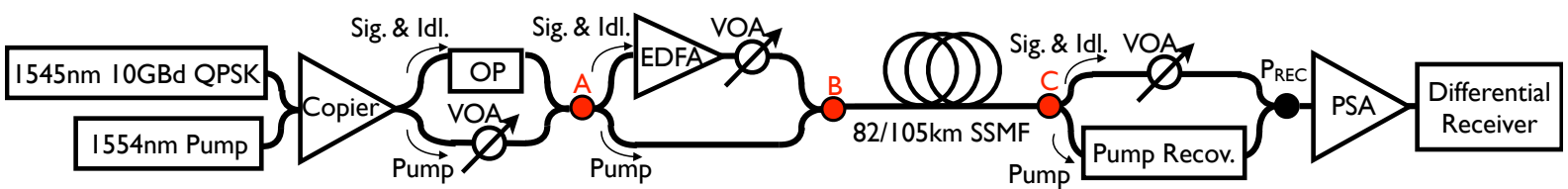

Fig. 3: Experimental set-up. Points $A-C$ indicate where dispersion compensation elements are added to the system. $\mathrm{P}_{\mathrm{REC}}$ denotes where received power is measured, VOA: variable optical attenuator. 
The insets in Fig. 4 show BER curves for $\mathrm{PI}$ and PS modes of operation for $0 \mathrm{dBm}$ signal launch power. In all cases PI to PS sensitivity gain is $>5 \mathrm{~dB}$, indicating good operation of the system. Fig. 4. shows $Q$ penalty, normalized to the highest measured $Q$ in each case, with $Q$ extracted from measured BER as $Q=20 \log _{10}\left[\sqrt{ } 2 \operatorname{erfc}^{-1}(2 B E R)\right]$. At low link input power, the received power is set such that the measured BER is close to $10^{-8}$. For PI FOPA operation, the penalty onset occurs at lowest launch power for the pre-compensation, higher for post- and slightly higher still for the optimized scheme. This is consistent with the EVM results from simulation. The PS case is noticeably more
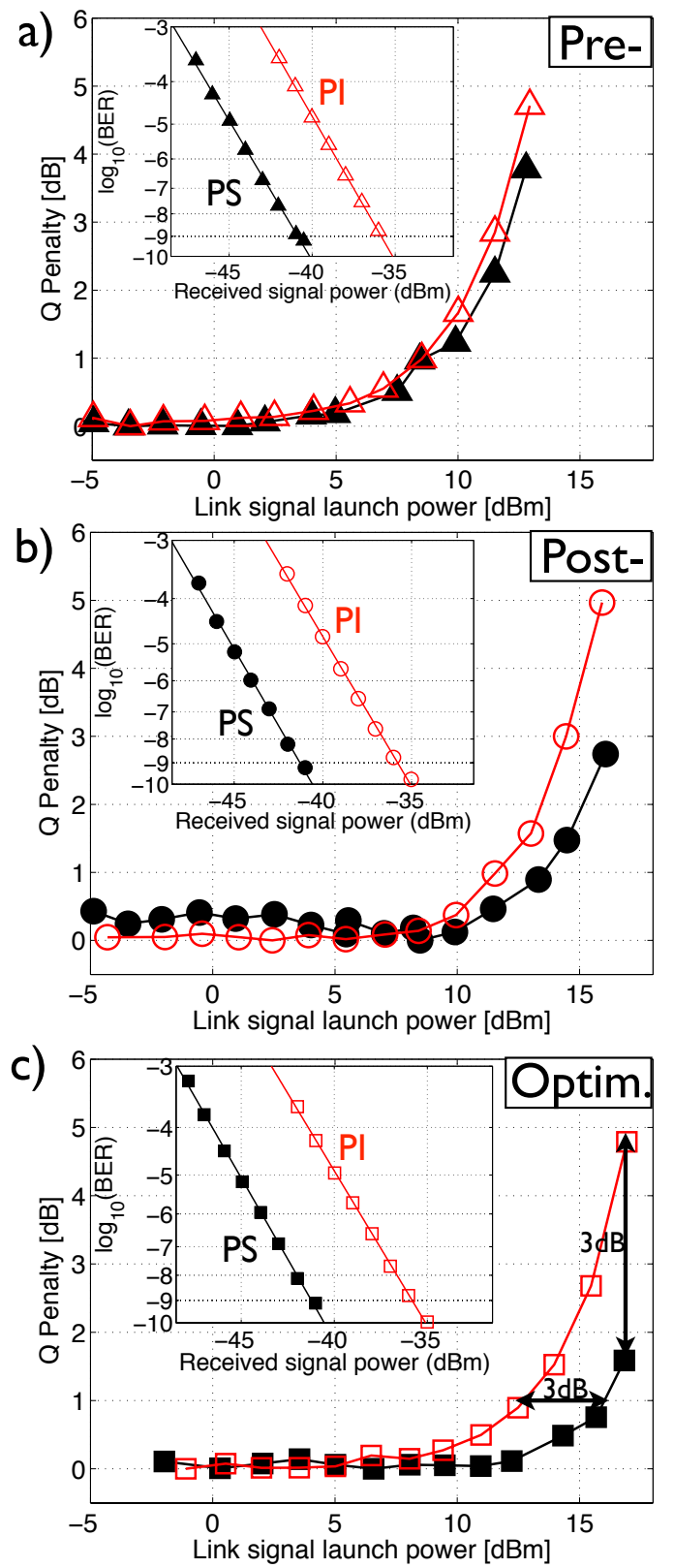

Fig. 4: $Q$ penalties for increased launch power. a)c) correspond to pre-, post- and optimal compensation schemes, respectively. Open red marks denote PI mode, solid black PS. (Inset BER curves in the linear regime) resistant to degradation than the $\mathrm{PI}$ case in all dispersion schemes. In the full pre- and postcases, maximum difference in $Q$ penalty is $2 d B$, similar to previous observations ${ }^{7}$. The optimized case improves upon this margin, increasing to $>3 \mathrm{~dB}$ over the range of powers measured. Moreover, if a penalty of $1 \mathrm{~dB}$ is tolerated, launch power can be increased by $3 \mathrm{~dB}$ in the optimized case (c.f. $2 \mathrm{~dB}$ for post-compensation). This confirms that tailoring the link dispersion map can indeed improve nonlinear phase noise mitigation, as suggested by simulations.

It is important to note that in this scheme, we utilize two wavelength channels, which has obvious implications for link capacity. However, as the sensitivity advantage of the PS system is demonstrably enhanced in the nonlinear regime over the PI system, this indicates potential for an overall increased capacity of these links ${ }^{10}$. Additionally, here we have investigated a singlehop link. The implications of the measured improvement are not clear for the use of this system in a multiple hop link, where the noise figure advantage of PSAs will also effect performance over many amplification stages. The flexibility of this system in mitigation of nonlinearities for WDM and higher-level modulation formats also remains to be studied.

\section{Conclusion}

We have shown that a copier-PSA link can provide mitigation of nonlinear distortions through the coherent superposition function of the PSA. Our results indicate that by engineering the link dispersion map, this effect can be enhanced. These results show the potential for copier-PSA systems to provide higher performance links not only through low noise figure amplification, but also by simultaneously mitigating penalties associated with high transmission power.

We thank OFS Denmark for the HNLFs used and Proximion $A B$ for the FBG DCMs. This work was supported by the ERC Advanced Grant PSOPA (291618), the Swedish Research Council (VR) and the Wallenberg foundation.

\section{References}

[1] Z. Tong et al., JSTQE, 10, 1016 (2012)

[2] K. Lee et al., Opt. Ex., 17, 20393 (2009)

[3] B. Corcoran et al., Proc. OFC'12, PDP5A.4 (2012)

[4] R. Slavik et al., Nat. Photon., 4, 690 (2010)

[5] T. Umeki et al., Proc. OFC'13, OW1I.7 (2013)

[6] Y. Tian et al., Opt. Ex., v, pp, (2013)

[7] S.L.I Olsson et al., Proc. ECOC'12, Th2F1 (2012)

[8] X. Liu et al., Proc. OFC'13, PDP5B.10, (2013)

[9] C. Lundström et al., PTL, 25, 234 (2013)

[10] C.J. McKinstrie et al., Opt. Ex., 19, 11977, (2011) 